\title{
¿Qué es la ciencia abierta?
}

\author{
What is open science?
}

\section{Lluís Anglada y Ernest Abadal}

Anglada, Lluís; Abadal, Ernest (2018). “¿Qué es la ciencia abierta?”. Anuario ThinkEPI, v. 12, pp. 292-298.

https://doi.org/10.3145/thinkepi.2018.43

Publicado en IweTe/ el 27 de febrero de 2018
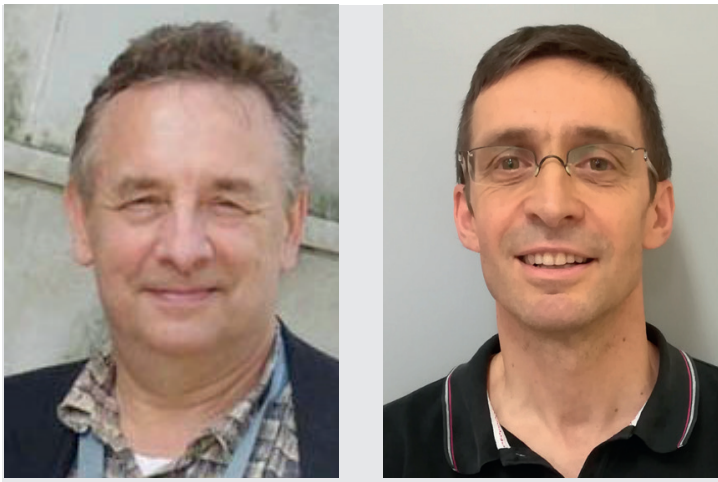

Resumen: Se analiza el concepto de ciencia abierta a partir del modelo de las revoluciones científicas de Thomas Kuhn: la ciencia abierta es un cambio de paradigma en la manera de llevar a cabo la investigación y, por tanto, supone modificaciones sustanciales en sus procedimientos. Se trata de un modelo promovido por la Comisión Europea sobre el cual se realizó una consulta pública. Los dos principales motivos para realizar este cambio son de tipo político-social (la riqueza de la sociedad se debe basar en la innovación) y también científicos. En esta nueva concepción, la ciencia debe ser abierta, colaborativa y hecha con y para la sociedad. Para explicar los elementos que forman parte de la ciencia abierta se han utilizado diversas metáforas. Los elementos siempre presentes en todas las representaciones son el acceso abierto, los datos abiertos, el open peer review y el software libre. También aparecen - aunque sin unanimidad- los recursos educativos abiertos, la ciencia ciudadana y los open notebooks. Finalmente, se incluyen algunas consideraciones sobre los cambios que comportará para los agentes de la investigación científica, en especial para los servicios bibliotecarios.

Palabras clave: Ciencia abierta; Comisión Europea; Paradigma científico; Investigación científica; Revolución científica.

Abstract: The concept of open science is analyzed using the model of scientific revolutions coined by Thomas Kuhn. In this way, open science is a paradigm shift in research and, therefore, involves substantial procedural modifications. It is a model promoted by the European Commission who gathered public opinion in Europe about it. The reasons for making this change are not only social (the wealth of society is based on innovation), but also scientific. In this new paradigm, science must be open, collaborative, and made with and for society. In order to explain the elements of open science, several metaphors are used in this article and always include open access, open data, open peer review, and open software. In some cases, open educational resources, citizen science, and open notebooks —although without unanimityare also included. Finally, we consider future changes within scientific research, especially as they relate to library services.

Keywords: Open science; European Commission; Scientific revolution; Paradigm shift; Research.

\section{Pero, ¿qué es esto de la ciencia abierta?}

Una buena manera de explicar qué es la ciencia abierta es a partir de la teoría de las revoluciones científicas que desarrolló el físico y filósofo de la ciencia Thomas Kuhn en su libro La estructura de las revoluciones científicas (Kuhn, 1962). Este autor llama "paradigmas" a:

"las realizaciones científicas universalmente reconocidas que, durante cierto tiempo, proporcionan modelos de problemas y soluciones a una comunidad científica".

Las tradiciones científicas y sus métodos serían maneras de pensar y de hacer que se mantendrían a 
pesar de la evolución de la ciencia y de sus prácticas. Un paradigma, una vez constituido, pervive en el tiempo a pesar de que dentro del mismo pervivan disfunciones o "anomalías". El proceso de sustitución de un paradigma "viejo" por uno nuevo es conflictivo debido al proceso emergente de nuevas prácticas y nuevas herramientas, así como a la inseguridad derivada del abandono de maneras de hacer bien asentadas en el tiempo.

\section{"La ciencia abierta es esto: un cambio de paradigma en la manera de hacer ciencia. El cambio no está en lo que se hace, sino en cómo se hace"}

La ciencia abierta es esto: un cambio de paradigma en la manera de hacer ciencia. Ésta no cambia sustancialmente con respecto a sus motivaciones y objetivos, pero sí lo hace (sustancialmente) en cuanto a sus métodos. El cambio no está en lo que se hace, sino en cómo se hace.

Una manera interesante de aproximarse a los contenidos de la ciencia abierta es utilizar la guía de recursos creada por Foster Plus (Fostering the practical implementation of open science in Horizon 2020 and beyond).

https://cordis.europa.eu/project/rcn/210056_es.html demanda para abordar los cambios sociales de nuestro tiempo. Tienen un impacto sobre todo el ciclo de la investigación, desde su inicio hasta la publicación de resultados, así como sobre la forma en la que se organiza este ciclo. Estos cambios se han denominado ciencia 2.0 o ciencia abierta" (European Comission, 2017a).

Estos cambios se aprecian muy bien en la figura 1, donde cada una de las fases del ciclo de la investigación está auxiliada por herramientas que no existían hace 5 o 10 años.

Lo que muestra la figura es ilustrativo, pero se queda en la superficie del cambio. La nueva forma de hacer ciencia quiere que ésta sea abierta, colaborativa, y hecha "con y para" la sociedad. Volveremos a estas ideas, pero demos un paso atrás para mencionar las motivaciones para abrazar este cambio, que son de dos tipos, las político-sociales y también científicas.

\section{Las motivaciones de Europa}

Las motivaciones político-sociales quedan bien explicadas con unas palabras de Jean-Claude Juncker, presidente de la Comisión Europea:

"La investigación y la innovación crean oportunidades de inversión para nuevos y mejores productos y servicios $y$, por tanto, incrementan la competitividad y el empleo" (European Comission, 2016, p. 5).
Éste es un proyecto de la Unión Europea que quiere ayudar a los investigadores a adoptar las diferentes prácticas de la ciencia abierta. Para ello, Foster Plus ha creado guías de recursos por disciplinas y el portal Foster, que da acceso a artículos e informes a través de una taxonomía.

https://www. fosteropenscience.eu

En el apartado 16 ("Ciencia con y para la sociedad") del programa Horizon 2020 de la Comisión Europea se indica:

"Hay cambios de largo alcance en el modus operandi del sistema científico, que son posibles por las tecnologías digitales y están impulsados por la globalización de la comunidad científica, así como por la creciente

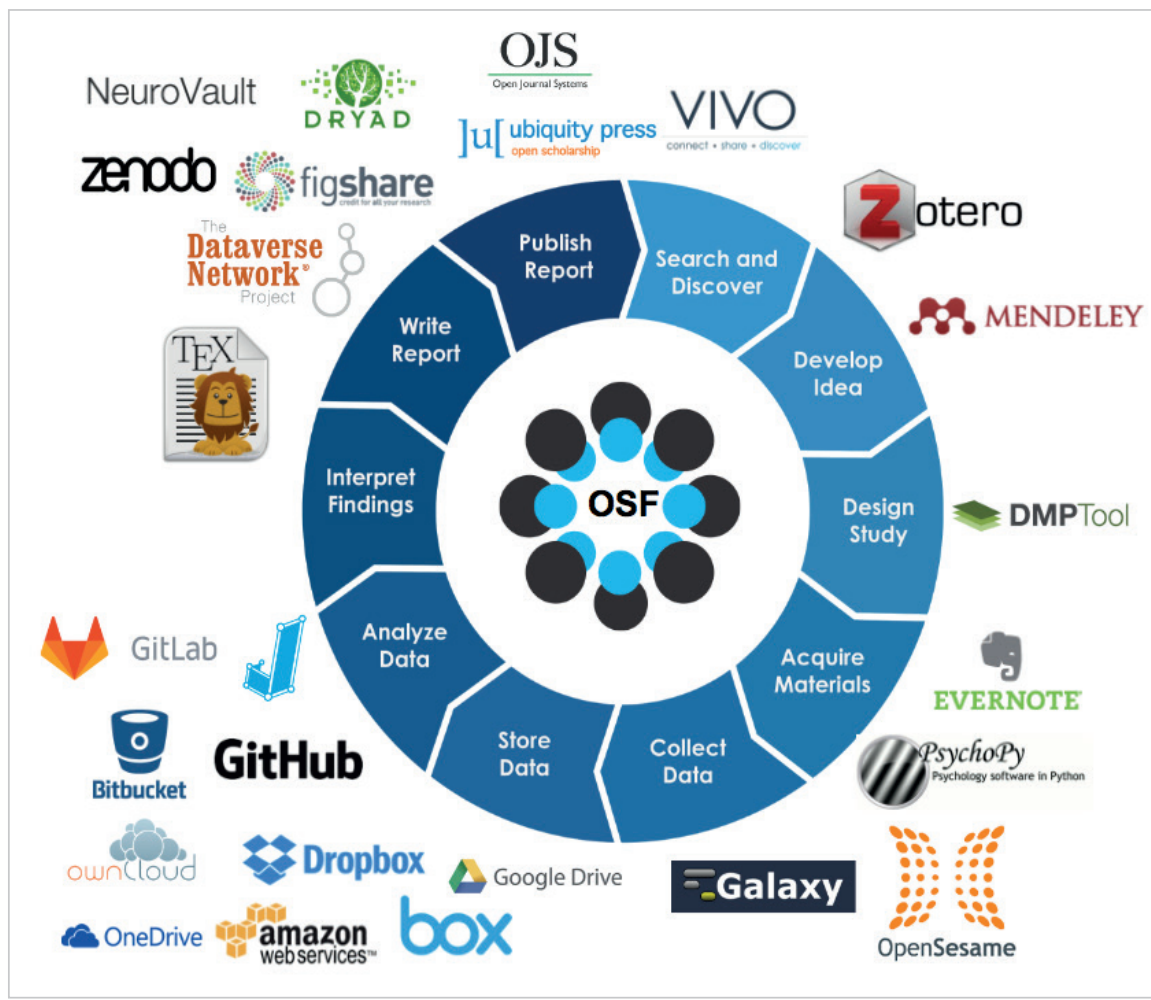

Figura 1. Fases de la investigación

Fuente: "The open science framework" Center for Open Science https://cos.io/our-products/osf 
Europa necesita mantener (y aumentar) sus niveles de bienestar y eso sólo lo puede hacer con una economía fuerte, que ya no puede basarse en la industria tradicional y que debe tener su centro en la innovación que se deriva de la investigación científica.

Pasemos a hablar de las motivaciones científicas. El movimiento de la ciencia abierta quiere adecuar la comunicación científica a las posibilidades tecnológicas actuales, quiere rediseñar el sistema de comunicación científica para reedificarlo con las herramientas de hoy, quiere aumentar su eficacia, es decir, eliminar las anomalías del sistema de difusión del conocimiento científico creado en un contexto tecnológico mecánico e impreso. El panorama del sistema actual está lleno de restricciones: revistas que sólo llegan a los suscriptores, artículos publicados mucho después de ser escritos, pasando filtros de calidad limitados a unos pocos revisores, sin incluir los datos en que se basan, etc. Restricciones que son -tecnológicamente hablando- obviables en el momento actual ya que la información digital se puede difundir de forma inmediata y universal por la Red.

Esta nueva manera de hacer ciencia -este nuevo paradigma - se fundamentaría en tres criterios: la ciencia debe ser abierta, colaborativa y hecha con y para la sociedad.

\section{"En el contexto de la ciencia abierta, lo que debe estar en abierto es cualquier resultado de la investigación (artículos y datos) así como los instrumentos auxiliares usados" \\ 3. Una ciencia abierta, colaborativa y hecha con y para la sociedad}

El acceso abierto a los contenidos académicos ha precedido al concepto de ciencia abierta $y_{\text {, }}$ quizá, ha condicionado algo su percepción. Si bien tenemos la tendencia de asimilar acceso abierto solamente a los artículos, en el contexto de la ciencia abierta, lo que debe estar en abierto es cualquier resultado de la investigación (artículos y datos) así como los instrumentos auxiliares usados, como pueden ser los cuadernos de laboratorio. Del mismo modo que lo era para artículos, aquí el significado de "abierto" es doble: gratuito y libre.

Mientras que la primera de las características de abierto requiere eliminar las barreras económicas para acceder a los resultados de la investigación, la segunda va más allá: se quiere que la ciencia hecha por uno o unos sea reutilizable por parte de cualquier otro. Una buena parte de la investigación, tal como se difunde hoy, encuentra muchos obstáculos para ser reproducida (o, en su caso falsada o comprobada) y esto se debe a que los artículos científicos no van acompañados de los datos en que se fundamentan ni de la descripción precisa de los procedimientos por los que se ha llegado. La ciencia abierta, al facilitar la reproducibilidad de las investigaciones, debe generar así mejor ciencia (Pontika et al., 2015, p. 1).

"Uno de los postulados base de la ciencia abierta es que la investigación debe ser hecha con y para la sociedad"

El incremento de colaboración entre los científicos es una de las tendencias de la ciencia moderna más claras de comprobar. En los últimos años, el número de autores por artículo ha crecido y lo ha hecho también la colaboración internacional (Dong et al., 2017). Pero la visión de la ciencia abierta va más allá de los ejemplos citados ya que concibe los retos científicos como interrogantes a resolver colectivamente Las posibilidades tecnológicas lo permiten, pero, además, y según la visión de la Comisión Europea, sólo la colaboración internacional entre científicos de todas las disciplinas puede permitirnos hacer frente a los grandes retos sociales de nuestro siglo (European Commission, 2013).

Aunque esté en la mente de todos, recordemos que el sustrato tecnológico que permite la colaboración científica ha incrementado de forma exponencial las posibilidades de compartir: el correo electrónico, plataformas en línea, redes sociales, comunicaciones virtuales... Si la ciencia moderna nació con el intercambio (primero de cartas entre científicos, más tarde publicando resultados en revistas), la ciencia futura será aún más colaborativa.

El último de los postulados base de la ciencia abierta es que la investigación debe ser hecha con y para la sociedad. Podemos decir que siempre ha sido así (o debe haber sido así), pero los documentos de la Comisión Europea ponen un acento especial en esto y lo desglosan en tres aspectos ${ }^{1}$ :

- lo que se ha venido en Ilamar ciencia ciudadana; - una ciencia responsable;

- una investigación científica dirigida a dar respuesta a los retos de la sociedad en el s. XXI.

Se reconoce implícitamente que la ciencia se ha desarrollado dentro de sus propios muros, con menor interacción que la debida con la sociedad que la financia y a la que se dirige o debe dirigirse. La solución que se ha usado hasta ahora ha 
sido la divulgación científica, solución que se ha mostrado insuficiente. La ciencia abierta observa que, en el contexto tecnológico actual, es posible establecer una nueva relación entre las personas y la ciencia, una relación de participación. Los ciudadanos no sólo pueden interesarse más por lo que hacen los científicos, sino que pueden contribuir a la ciencia con su participación².

\section{"En el contexto tecnológico actual, los ciudadanos no sólo pueden interesarse más por lo que hacen los científicos, sino que pueden contribuir a la ciencia con su participación"}

\section{La interesante gestación del término}

El término "ciencia abierta" ha tenido una vida feliz pero su gestación ha sido larga. Hoy en día es un concepto ampliamente utilizado, aunque con diferente intensidad según los lares en que se habite. Su mayor uso en Europa no es casual porque ha sido asumido como programa por la Comisión Europea, y su mucho menor uso en los Estados Unidos de América es indicativo de las diferencias significativas en el enfoque de este momento de transición.

En marzo de 2013, la Dirección General de Communications Networks, Content and Technology (Connect) de la Comisión Europea, en consonancia con uno de sus objetivos prioritarios (el del mercado único digital), publica un documento conceptual sobre el momento actual de la ciencia (European Commission, 2013). En él se exponen de forma embrionaria los conceptos que la Comisión ya no dejará de usar en documentos posteriores: internet y otras tecnologías han cambiado radicalmente la forma en que se crea y difunde el nuevo conocimiento, este cambio necesita sustentarse en un entorno científico colaborativo y abierto, y la nueva manera de hacer ciencia permite que esta se acerque a la sociedad (European Commission, 2013, p. 1). En el resto del documento se muestran los elementos constitutivos de esta forma nueva de ser de la ciencia, se exponen sus potencialidades (para la misma ciencia y para la sociedad), y se sugieren acciones de futuro relacionadas con el programa Horizon $2020^{3}$.

Debido al impacto que la Comisión intuye que tiene el desarrollo del concepto de ciencia abierta para la actividad de todos los actores implicados en el ciclo de investigación, organizó una consulta sobre la ciencia en transición. La consulta partía de un documento base el que se explicaba el concepto de (la entonces llamada) ciencia 2.0, sus implicaciones, las oportunidades que presentaba, las barreras a las que hacía frente, y finalizaba con un análisis del papel que tenían que tener las agencias financiadoras de la investigación, los estados y la Unión Europea para facilitar su adopción (European Commission, 2014). Las medidas se orientaban al acceso abierto de publicaciones (ya obligatorio entonces para los proyectos financiados por Europa) y datos (iniciado de forma piloto).

\section{Los componentes}

Existen diversas interpretaciones sobre cuáles son los elementos que forman parte de la ciencia abierta. Cada una de ellas, además, acostumbra a utilizar una metáfora para explicar su interrelación: los ejes de la rueda, los pilares, las celdas del panal o el ciclo de la investigación. Vamos a comentarlas a continuación ordenándolas según el número de componentes de la ciencia abierta que se consideran.

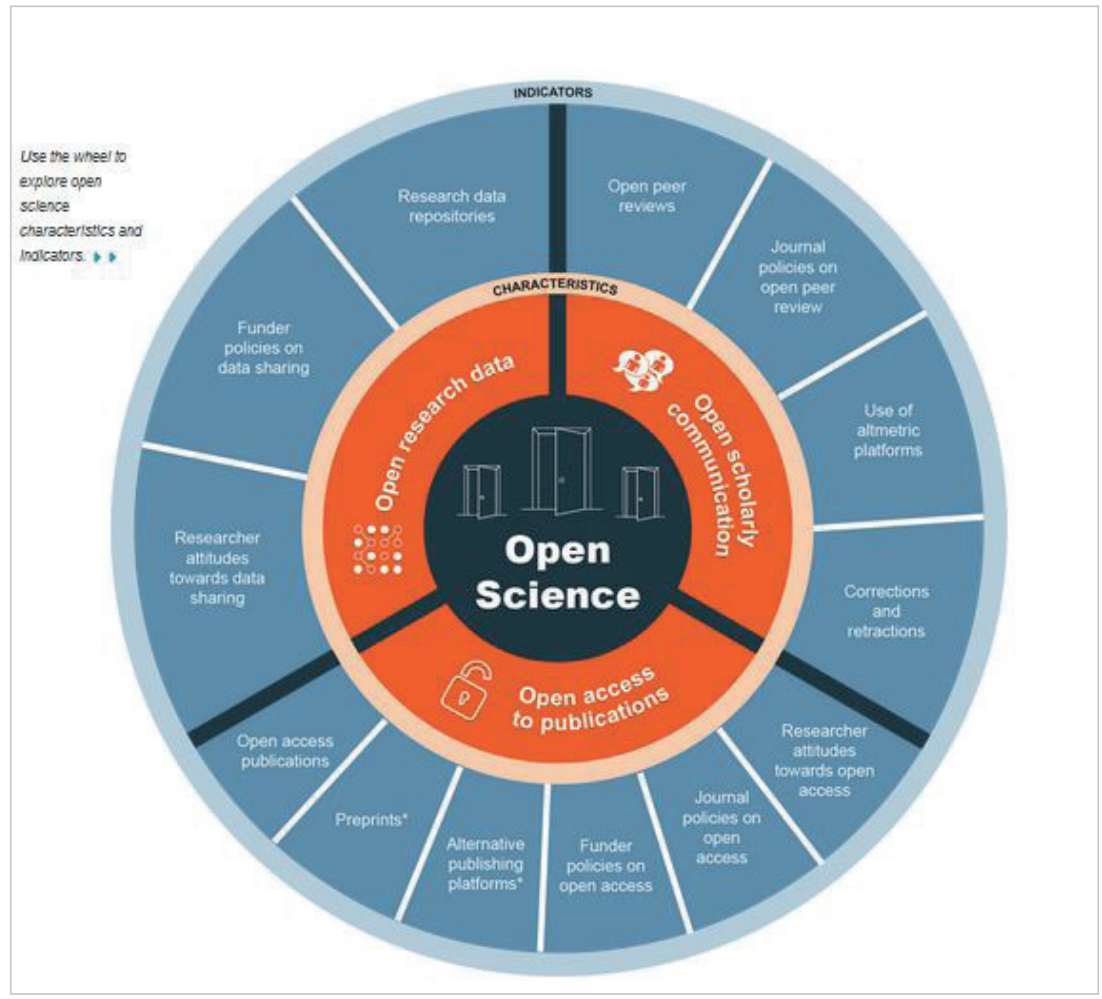

Fig. 2. Los ejes de la ciencia abierta (European Comission, 2017b) 
El proyecto Open Science Monitor (European Comission, 2017b) utiliza la metáfora de los ejes que hacen girar la rueda de la ciencia abierta en la cual se consideran tres ejes o elementos básicos:

- datos abiertos de investigación;

- acceso abierto;

- comunicación científica abierta.

Masuzzo y Martens (2017), por su parte, utilizan la metáfora de los pilares o columnas que sustentan la ciencia abierta que, en su opinión, serían cuatro:

- datos abiertos;

- código abierto;

- open access;

- revisión abierta.

El proyecto Foster, que dispone de uno de los portales más completos y detallados sobre ciencia abierta, describe los componentes de la ciencia abierta como las celdas de un panal de abejas. Incluye aquí ocho elementos que se van ajustando para formar la estructura del panal de la ciencia abierta:

- open notebooks;

- datos abiertos;

- revisión abierta;

- open access;

- sotfware libre;

- redes sociales académicas;

- ciencia ciudadana;

- recursos educativos abiertos.

En esta lista constan los cuatro elementos anteriores y se le suman otros cuatro.

Finalmente, existe también la metáfora del ciclo de vida o de las fases de la investigación, que podemos encontrar en el informe RIN \& Nesta (2010), en Szkuta y Osimo (2012) y tam-

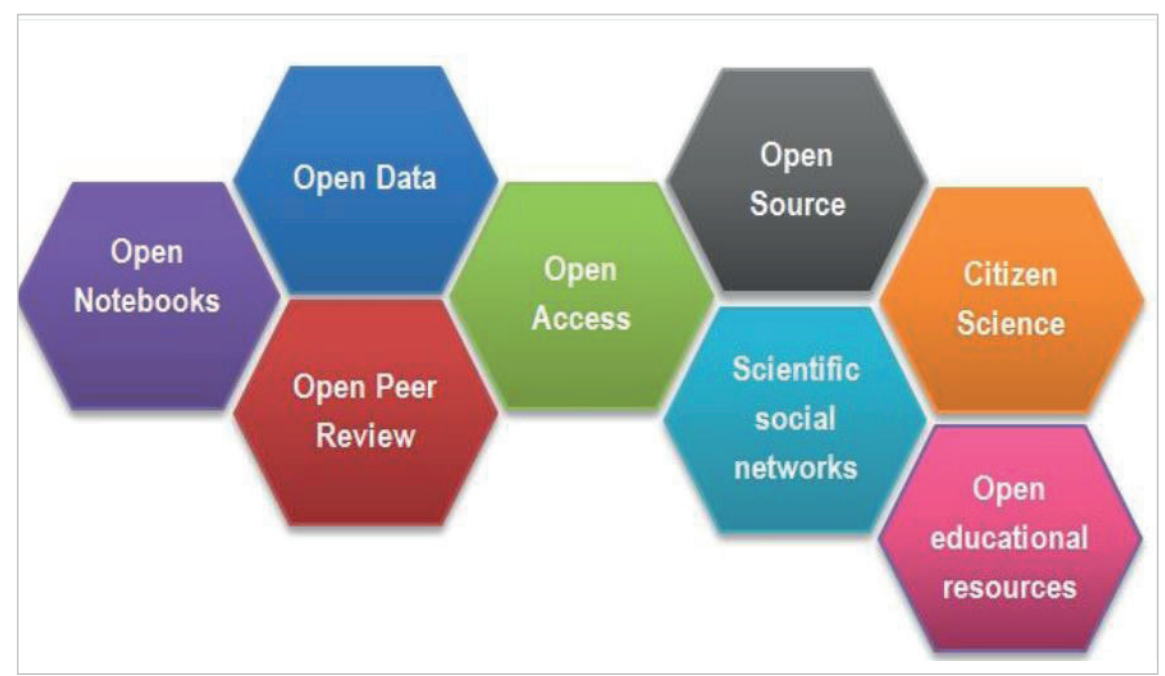

Fig. 4. Las celdas del panal de la ciencia abierta (Foster)

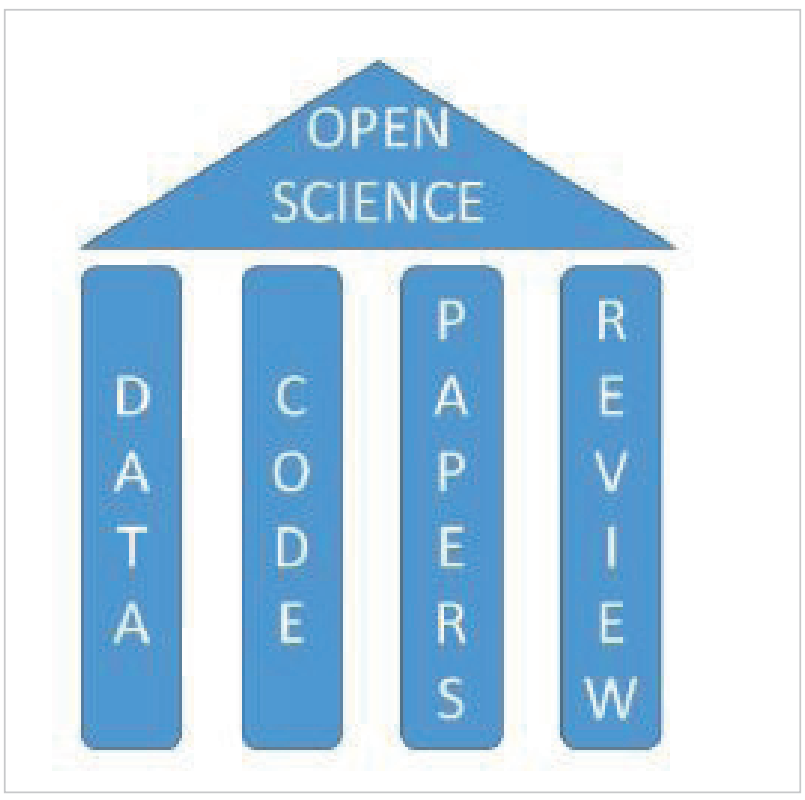

Fig. 3. Los pilares de la ciencia abierta (Masuzzo; Martens, 2017)

bién en el propio Foster. Anteriormente hemos comentado que la ciencia abierta es una nueva manera de abordar la investigación científica. Esta representación que ahora comentamos intenta explicar cómo se puede llevar a cabo y ejecutar la investigación en el marco de la ciencia abierta. En este caso, se contempla el diseño, la metodología, la recogida de datos, la revisión, la publicación, la difusión, etc. cada una de ellas con el enfoque que le corresponde de la ciencia abierta, ya sea el open data, open peer review, open access, etc.

Existen, por tanto, diversas conceptualizaciones o metáforas para explicar los elementos que forman parte de la ciencia abierta, así como una diversidad de componentes para cada uno de los modelos.

Los elementos siempre presentes en todas las representaciones son el acceso abierto, los datos abiertos, el open peer review y el software libre. En un segundo bloque también aparecen (aunque sin tanta unanimidad) los recursos educativos abiertos, la ciencia ciudadana y los open notebooks. Todos ellos merecen un análisis más detenido que no podemos abordar en este texto. 


\section{Implicaciones}

Está claro que esta manera nueva de hacer ciencia supondrá cambios importantes para todos los agentes de la investigación científica. Para los investigadores en primer lugar, pero también para las agencias de financiación de la investigación, los organismos y procesos de evaluación, los gestores de programas y proyectos de investigación y la ciudadanía en general.

También supondrá cambios destacables en los servicios bibliotecarios. Las bibliotecas tienen una larga tradición de apoyo a los investigadores, pero las actividades que han Ilevado a cabo para hacerlo son las que surgieron como más adecuadas en un panorama que ahora está cambiando rápidamente. En la era de lo impreso, no el único, pero sí el principal apoyo de las bibliotecas a la investigación consistió en facilitar el acceso a la información científica con la construcción de colecciones propias y el suministro de documentos.

En el marco de la ciencia abierta - sobre todo cuando ya esté consolidada- los problemas se redefinen. La accesibilidad a la información será una característica en buena medida intrínseca a la misma información, pero los componentes de la actividad científica ya no se limitan a los artículos de las revistas, ya que incluyen los datos y los cuadernos de laboratorio, por ejemplo, y su gestión se hace más compleja.

En el marco de la ciencia abierta la investigación requiere de muchos servicios de apoyo. Apoyo para descubrir información, apoyo para publicarla de forma abierta y complementada con datos, apoyo para difundirla a través de redes sociales y entre la sociedad, etc. En este marco cambiante, y aún no totalmente definido, las bibliotecas deberán desarrollar sus servicios y encontrar la forma de hacerlo no será una dificultad menor.

\section{Notas}

1. Otros conceptos incluidos en el de ciencia con y para la sociedad son: la educación científica, la igualdad de género, el uso de información científica por parte de los ciudadanos, la ética de la ciencia...

Ver los planes de trabajo del programa Horizon 2020 de la Comisión Europea https://ec.europa.eu/programmes/horizon2020/en/ h2020-section/science-and-society

2. Un ejemplo que nos permite entender el concepto es la plataforma eBird que permite a ciudadanos de a pie aficionados a avistar pájaros y sus migraciones anoten sus observaciones para crear así un conjunto de datos que no sería posible recoger de forma alternativa. http://ebird.org

3. En al anexo 1 del documento se hace un interesante repaso del concepto digital science y sus adyacentes. Nótese los conceptos analizados: e-Science, open science, science 2.0 y digital science.

\section{Bibliografía}

Dong, Yuxiao; Ma, Hao; Shen, Zhihong; Wang, Kuansan (2017). A century of science: Globalization of scientific collaborations, citations, and innovations. ArXiv:1704.05150v1

https://arxiv.org/abs/1704.05150

European Commission (2013). Digital science in Horizon 2020. March.

https://ec.europa.eu/digital-sing/e-market/en/news/ digital-science-horizon-2020

European Commission (2014). Public consultation: 'Science 2.0': Science in transition (background document). Directorates-General for Research and Innovation (RTD) and Communications Networks, Content and Technology (CONNECT).

https://ec.europa.eu/research/consultations/science-2.0/ background.pdf

European Comission (2016). Open innovation, open science, open to the world: A vision for Europe. Brussels: European Commission. Directorate-General for Research and Innovation. 
https://publications. europa.eu/s/fzsT

European Comission (2017a). Horizon 2020: Work programme 2016-2017. 16. Science with and for society. European Commission Decision C(2017) 2468 of 24 April 2017.

http://ec.europa.eu/research/participants/data/ref/ h2020/wp/2016_2017/main/h2020-wp1617-swfs_en.pdf

European Comission (2017b). Open science monitor. https://ec.europa.eu/research/openscience/index. cfm?pg=home\&section=monitor

Foster (2018). The future of science is open. https://www.fosteropenscience.eu/

Kuhn, Thomas S. (1962). The structure of scientific revolutions. Chicago: University of Chicago Press. ISBN: 0 226458040

https://goo.gl/MUi1i3

Masuzzo, Paola; Martens, Lennart (2017). “Do you speak open science? Resources and tips to learn the language". PeerJ Preprints.

http://oro.open.ac.uk/44719/

https://doi.org/10.7287/peerj.preprints.2689v1

Pontika, Nancy; Knoth, Petr; Cancellieri, Matteo; Pearce, Samuel (2015). "Fostering open science to re- search using a taxonomy and an eLearning portal". En: iKnow: 15th International Conference on Knowledge Technologies and Data Driven Business, 21-22 October 2015, Graz, Austria. ISBN: 9781450337212

http://oro.open.ac.uk/44719 https://doi.org/10.1145/2809563.2809571

RIN; Nesta (2010). Open to all? Case studies of openness in research: A joint RININESTA report.

http://www.rin.ac.uk/system/files/attachments/NESTARIN_Open_Science_V01_0.pdf

Szkuta, Katarzyna; Osimo, David (2012). "Openess in the research cycle". En: Science 2.0 study, 29 mayo. https://science20study.wordpress.com/2012/05/29/ openness-in-the-research-cycle

Lluís Anglada

CSUC, Área de Ciencia Abierta Iluis.anglada@csuc.cat

Ernest Abadal

Universitat de Barcelona

Facultat de Biblioteconomia i Documentació abadal@ub.edu

\section{No descuides mantener actualizados tus datos en el Directorio EXIT}

\section{(EXpertos en el Tratamiento de la Información)}

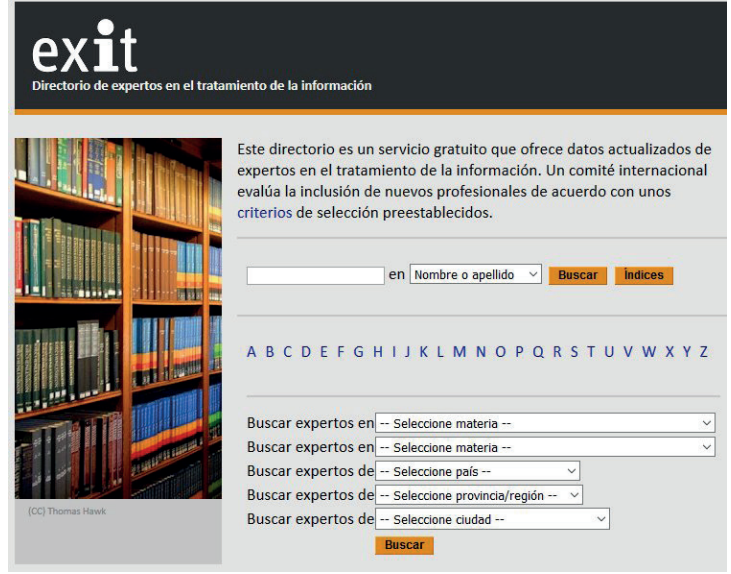

Países

España Colombia

México

Brasil

Argentina

Reino Unido

Chile

Cuba

EUA

Perú

Ecuador

Italia

Portugal

Francia

Holanda

Alemania

Venezuela

Bélgica

Uruguay

Suiza $\triangle$ curriculum

e. LiS 6 documents

8 Google Citations

\$. Mendeley

RG ResearchGate

[R] ResearcherID

(iD) Orcid

इ' Google+

f Facebook

v. Twitter
Puedes incluir enlaces a tus páginas de las redes sociales

Especialidades más representadas en EXIT no

Gestión de la información y del conocimiento

Web 2.0 y redes sociales

Biblioteca universitaria

Comunicación

Recuperación de información y búsquedas

Información científico-técnica

Biblioteca digital

Planificación y gestión

Educación, formación, alfin

Gestión de contenidos

Análisis y diseño de sistemas de información

Open access

Bibl. especializada / Centro de documentación

Catalogación, clasificación e indexación

Bases de datos (contenidos)

Bibliometría y cibermetría

Marketing y promoción

Periodismo

Revistas electrónicas

Documentación en general

Arquitectura de la información

Información biomédica

Diseño

Servicios de internet en general

Usabilidad, interfaces e interacción

Biblioteca pública

Multimedia y documentación audiovisual

Información humanística

Software documental

Ontologías, metadatos, taxonomías y tesauros
1.056

924

784

733

678

654

563

524

489

461

460

457

449

409

403

398

360

337

324

311

309

307

287

282

266

249 